\section{Category}

Synthesis of Natural

Products and

Potential Drugs

\section{Key words}

sarpagine alkaloids

peraksine

haloboration

palladium catalysis

vinylation
R. V. EDWANKAR, C. R. EDWANKAR, J. R. DESCHAMPS, J. M. COOK* (UNIVERSITY OF WISCONSIN-MILWAUKEE AND NAVAL RESEARCH LABORATORY, WASHINGTON, D.C., USA)

General Strategy for Synthesis of C-19 Methyl-Substituted Sarpagine/Macroline/Ajmaline Indole Alkaloids Including Total Synthesis of 19(S),20(R)-Dihydroperaksine, 19(S),20(R)-Dihydroperaksine-17-al, and Peraksine J. Org. Chem. 2014, 79, 10030-10048.

\title{
Total Synthesis of (+)-Dihydroperaksine-17-al, (+)-Dihydroperaksine, and (+)-Peraksine
}
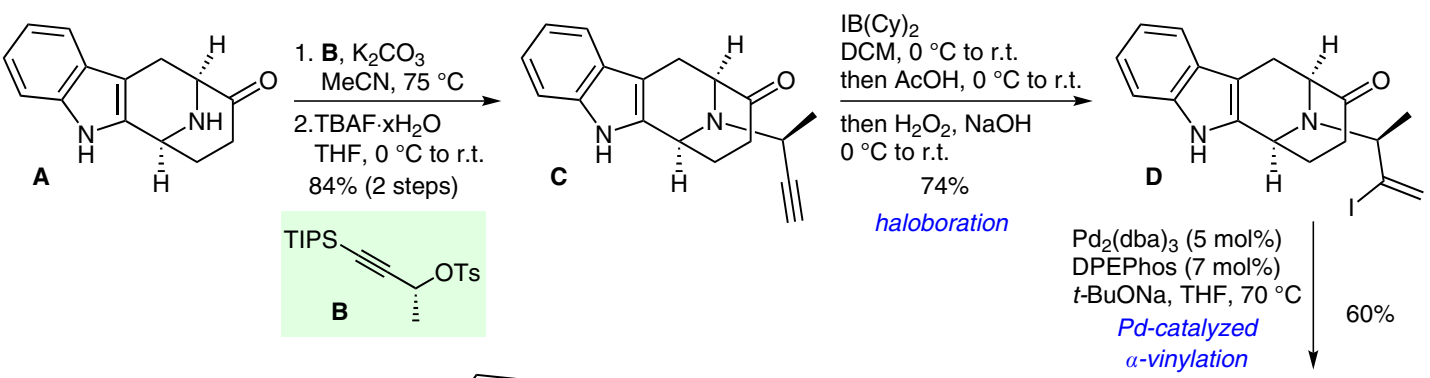

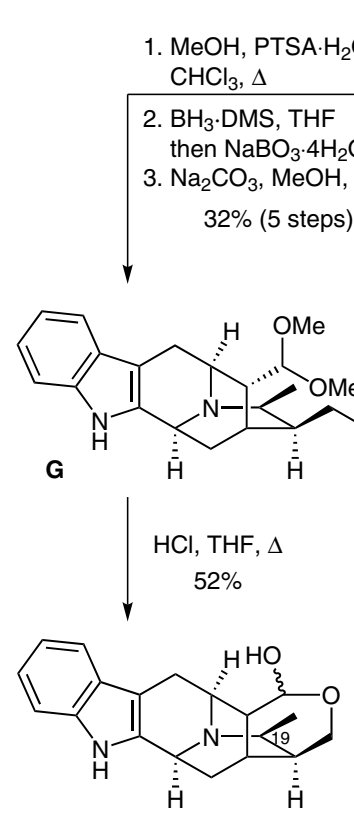

(+)-Peraksine
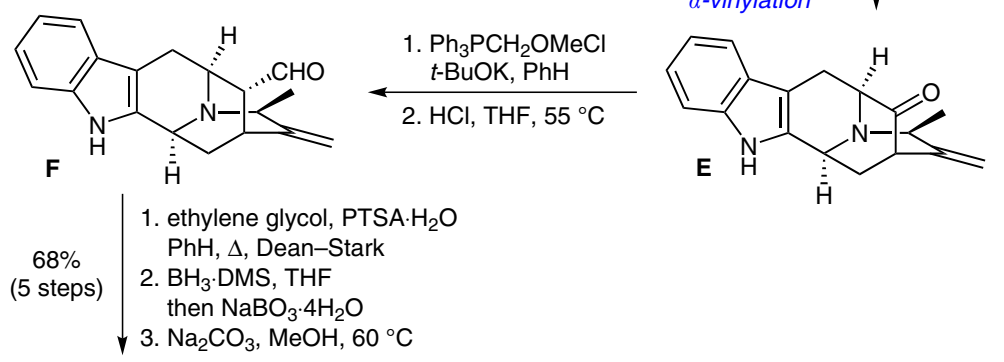

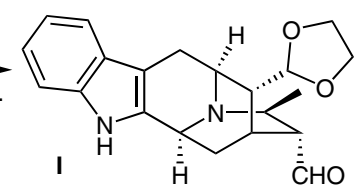

H

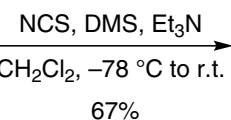

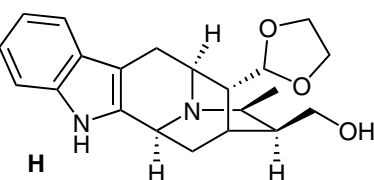

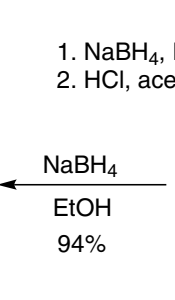

(+)-Dihydroperaksine

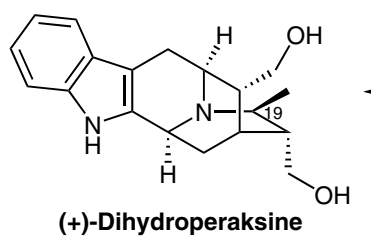

Significance: The sarpagine alkaloids

(+)-19(S),20(R)-dihydroperaksine-17-al,

(+)-19(S),20(R)-dihydropraksine (both isolated from Rauwolfia serpentina) and (+)-peraksine (isolated from Rauwolfia perakensis) have in common the structural feature of a $\beta$-methyl group at C-19. Cook and co-workers report the first enantio- and stereospecific synthesis of all three alkaloids.
Comment: After introduction of the chiral methyl group by $\mathrm{N}$-alkylation, the pentacyclic core was formed by haloboration followed by a palladiumcatalyzed intramolecular $\alpha$-vinylation of the ketone. Common intermediate $\mathbf{F}$ was then converted into (+)-peraksine, (+)-dihydroperaksine-17-al, and (+)-dihydropraksine by a specific acetal protection and hydroboration-oxidation sequence.

SYNFACTS Contributors: Erick M. Carreira, Adrien Joliton 